\title{
The Role of Nanomorphology and Interfacial Structure of Platinum Nanoparticles in Catalysing the Hydrogen Oxidation Reaction
}

Xue Jiao, Christopher Batchelor-McAuley, Chuhong Lin, Enno Kätelhön, Eden E.L. Tanner ${ }^{\dagger}$, Neil P. Young ${ }^{\ddagger}$ and Richard G. Compton*

${ }^{\dagger}$ now at the School of Engineering \& Applied Sciences, Harvard University, Cambridge, MA, USA

‡ Department of Materials, Oxford University, 16 Parks Road, Oxford, OX1 3PH, United Kingdom

* corresponding author: Richard G. Compton, Department of Chemistry, Physical \& Theoretical Chemistry Laboratory, Oxford University, South Parks Road, Oxford, OX1 3QZ, United Kingdom Email: richard.compton@chem.ox.ac.uk. Tel: +44(0)1865275957 Fax: +44(0)1865275410

\begin{abstract}
This work studies the hydrogen oxidation reaction using both single mesoporous platinum nanoparticles $\left(\mathrm{r}_{\mathrm{np}}=23.1 \pm 2.1 \mathrm{~nm}\right)$ and low density random arrays of material. The activity of the platinum catalyst, towards the oxidation of hydrogen, is shown to be potential dependent and exhibits two peaks in activity. These peaks in activity are measured at both random arrays of platinum nanoparticles and at the single entity scale. This alteration in the particle activity is directly reflected in the variation of the electrochemical current. These peaks in current do not relate to a mass-transport limitation of the reaction and at high overpotentials the oxidation reaction becomes fully inhibited. This potential dependency is revealed at high current densities and arises due to the sensitivity of the reaction rate to the platinum interfacial structure; the decrease in activity (at ca. $-0.2 \mathrm{~V}$ vs $\mathrm{Ag} / \mathrm{AgCl}(1 \mathrm{M} \mathrm{KCl})$ in non-buffered condition) directly correlates with the potential at which underpotential deposited hydrogen is removed from the catalytic interface. The contribution of the internal mesoporous nanoparticle structure towards the total particle catalytic activity is further evidenced through comparison of the time-current transients recorded for individual nanoparticles of differing morphology (solid versus mesoporous) and by evidencing the sensitivity of the single particle catalytic activity to the used supporting electrolyte concentration.
\end{abstract}

Keywords: catalysis, electrochemistry, hydrogen, platinum nanoparticles, surface chemistry 


\section{Introduction}

With the advent of energy conversion technologies such as fuel cells, redox flow batteries and solar cells the development of advanced electrocatalytic materials has been a significant focus. However, for a heterogeneous process at a solid-liquid interface, quantifying the efficiency of a given catalytic material is often complicated by the difficulty in clearly delineating the contributions from the chemical and physical aspects of the material; altering a surface's architecture ${ }^{1}$ and morphology may vary the number and/or nature of the active sites, change the local mass-transport or in the case of a redox reaction cause the local solution potential profile to be altered. Development of new materials beyond recourse to empiricism requires the study of archetypal reactions under welldefined conditions to enable the development of greater theoretical insight. Over the preceding century our understanding of outer-sphere redox reactions has advanced massively with seminal contributions from Marcus ${ }^{2}$, Hush $^{3-4}$ and in relation to electrochemical interfaces Chidsey. ${ }^{5}$ In contrast, plausibly partially hindered by a lack of experimental information, our theoretical understanding of so-called inner-sphere reactions, proceeding via surface adsorbed intermediates, is arguably still relatively limited.

For a heterogeneous redox reaction the electrochemical 'reversibility' of the process is controlled by the interfacial rate constant relative to the local mass-transport. Consequently, studying the kinetics of fast interfacial processes necessitates the use of high mass-transport conditions. One route by which such high rates of mass-transport may be attained is though the study of interfacial reactions at individual nanoparticles. ${ }^{6-7}$ For many conventional experiments the influence of the double layer upon the mass-transport of solution phase material can (to a reasonable approximation due to the differing length scales involved) be ignored. ${ }^{8}$ However, as experiments move from studying electrochemical reactions at the micro to the nanoscopic scale the diffusional depletion zone contracts and the two processes cannot be considered fully independent. ${ }^{9}$ As a further complicating factor interfacial redox processes proceed via surface intermediates, these species are necessarily situated within the so-called inner-Helmholtz plane and at such length scales the double layer structure, although considered at great length, remains essentially experimentally unvalidated. ${ }^{10}$

The proton/hydrogen redox couple is a paradigmatic example of an inner-sphere interfacial process and it is upon this reaction that much of our insight into this reaction class is gained. At a platinum surface the kinetics of the hydrogen/proton redox couple are 'fast' with the electron transfer being near 'reversible' in acid ${ }^{11}$ and slower in alkaline media with the reaction rate exhibiting sensitivity to the surface crystallinity. ${ }^{12}$ This reaction still holds information regarding the processes occurring 
at the ångström length scale with recent work by Koper et al. demonstrating the likely importance of the local interfacial water structure in controlling the reaction rate. ${ }^{13}$ Under alkaline conditions the addition of a sub monolayer coverage of $\mathrm{Ni}(\mathrm{OH})_{2}$ was found to appreciably alter and increase the kinetics of this process. The influence of the nickel was interpreted as due to a change in the potential of zero free charge, hence influencing the 'rigidity' of the water situated at the interface. At higher overpotentials and under high mass-transport conditions, it was recognised as early as the 1950s by Frumkin ${ }^{14}$ that, even on platinum, the hydrogen oxidation reaction (HOR) becomes limited by the rate of chemi-sorption of hydrogen onto platinum surfaces. Recent work has demonstrated that in this adsorption limited regime the current is not constant and exhibits two peaks in the activity of the catalyst as a function of potential. The origin of this so-called 'fine structure' is debatable but has been previously described using a two site model. ${ }^{15}$ A significant focus of this work will be to further elucidate the physical origins of this variation in activity and evidence that this variability can be observed at the single particle scale.

Apart from being of fundamental importance the hydrogen/proton redox couple is key to the possible realisation of the hydrogen economy. Advances in fuel cells have led to a dramatic lowering of the required platinum loading, with the present state of the art requiring $\sim 0.25 \mathrm{~g}$ of platinum group metal per kilo Watt of power. ${ }^{16}$ As a consequence of this decrease in the platinum loading and the operation of these devices at high currents - in part to afford greater power - large turnover rates occur at the surface of the catalyst, with current densities in the order of $1 \mathrm{~A} \mathrm{~cm}^{-2}{ }^{17}$ Consequently, there is a distinct need to study well characterised catalyst materials under conditions of high current density. ${ }^{18}$ Single particle studies represent an extreme limit for the investigation of low particle loadings and ensure high rates of mass-transport to the catalytic interface; ideal for the investigation of fast electrochemical processes. Traditionally electrocatalytic materials are characterised through the use of a rotating disc electrode and it is by this methodology that all new fuel cell catalysts are benchmarked. ${ }^{19-21}$ For commercial rotating disc electrode (RDE) devices the maximum rotation rate is commonly in the order of $10^{4} \mathrm{rpm} .{ }^{22}$ At these speeds the diffusion limited current for a molecular species in millimolar concentration is limited to a few $\mathrm{mA} \mathrm{cm}^{-2}$ and corresponds to a diffusional mass-transport rate of $\sim 1 \times 10^{-3} \mathrm{~m} \mathrm{~s}^{-1}$. The reporting of higher 'kinetic' current densities is often achieved through the use of the Koutecky-Levich equation allowing, under optimal conditions, for the mass-transport limitation to be accounted for. For heterogeneous surfaces the validity of such an approach may be questioned. ${ }^{23-25}$ Importantly, the low current electrocatalyst characteristics as derived from RDE type experiments do not necessarily correlate in a simple manner with whole device performance, ${ }^{18}$ where in real applications the catalytic material is utilised under extreme current densities to ensure efficient and optimal use of the platinum group 
metals. Decreased catalyst loading at an RDE is one route by which higher specific current densities may be experimentally attained; however, with low catalyst loadings, complications arise in the production of reproducible and relatively homogeneous nanoparticle surface coverages. ${ }^{19}$ Alternately fabrication of macroscopic gas-diffusion electrodes allows material to be studied under high-current densities; ${ }^{26}$ however, the mass-transport profile of such systems is poorly defined due to the need to account rigorously for the structure and tortuosity of the porous gas membrane. Single nanoparticle studies provide a complementary route for the fundamental investigation of interfacial reactions under high mass-transport conditions.

Previous work looking at the response of individual mesoporous platinum nanoparticles have emphasised electrochemical accessibility of the internal interfaces towards the formation of surface oxides $^{27}$ and have demonstrated the ability of HOR and HER to be studied on the single particle scale. ${ }^{28-30}$ This work studies the hydrogen oxidation reaction at individual mesoporous nanoparticles revealing how the nanoparticle structure contributes to the catalytic performance of the material. The study of single nanoparticle reactions has been partially hampered by colloidal stability in the presence of electrolytes. ${ }^{31-32}$ However, since the 1980s it has been recognised that use of a microelectrode enables redox reactions to be driven at an electrochemical interface in the absence of high electrolyte concentrations and without significant ohmic distortion. ${ }^{33-34}$ This, as will be demonstrated, holds true at the nanoscale where due to the small currents involved (picoampere) redox reactions can be performed at individual nanoparticles in resistive media and importantly allow insight into the activity of the mesoscale nanoparticle structure.

This work investigates the influence of both the chemical (interfacial structure) and physical (nanoparticle mesoporous structure) factors contributing and controlling the activity of a platinum catalyst towards the oxidation of hydrogen. Both random arrays of particles and individual platinum entities show potential dependent variability in the catalytic activity of the material. However, studying the HOR on a single particle basis enables the reaction to be studied under conditions of very high mass-transport (approaching $0.15 \mathrm{~m} \mathrm{~s}^{-1}$ for a completely isolated $23 \mathrm{~nm}$ radius particle). Under these high current density conditions it is revealed that even at the single particle scale there are peaks in the catalytic activity, as reflected in the measured electrochemical current of the platinum catalyst, varying as a function of the applied potential. These peaks relate not to a masstransport limitation but reflect the change and decrease of the interfacial reaction rate at high overpotentials. Moreover, by studying single catalytic nanoparticles one at a time the influence of the nanoparticles internal mesoporous structure upon the overall reaction rate is exemplified. 


\section{Experimental}

\subsection{Chemicals}

Hydrogen $\left(\geqslant 99.98 \% \mathrm{H}_{2}\right)$ was supplied from BOC, Surrey, U.K. Potassium nitrate ( $\geqslant 99.0 \%$, $\mathrm{KNO}_{3}$ ), Potassium perchlorate ( $\left.\geqslant 99 \%\right)$, and perchloric acid (70\%) were obtained from SigmaAldrich, Dorset, U.K. Potassium hydroxide were obtained from Fisher Scientific, Loughborough, U.K. Citrate-capped mesoporous platinum nanoparticles (mesoporous PtNPs) were provided by nanoComposix, San Diego, CA, U.S.A., with a reported diameter $50 \mathrm{~nm}$ and concentration $3.3 \times$ $10^{13}$ particles $\mathrm{L}^{-1}$. Solid platinum nanoparticles (solid PtNPs) were provided by Particular GmbH,

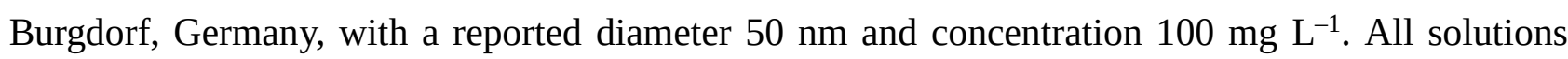
were prepared with ultrapure water from Millipore, with a resistivity of not less than $18.2 \mathrm{M} \Omega \mathrm{cm}$ at $298 \mathrm{~K}$.

\subsection{Electrochemistry}

\subsubsection{Macro-electrode and Drop-Cast Experiments}

A three-electrode system in a Faraday cage was used for all ensemble electrochemical experiments. A $\mu$ Autolab III from Metrohm Autolab B.V., Utrecht, The Netherlands, was employed as a potentiostat. The working electrode was a platinum or gold macrodisk electrode of radius $831.5 \mu \mathrm{m}$ or $1 \mathrm{~nm}$ from BASi, West Lafayette, IN, U.S.A., and the electrode was polished with alumina powders from Buehler, Lake Bluff, IL, U.S.A., in a size sequence of 1.0, 0.3, and $0.05 \mu \mathrm{m}$. The reference electrode was a standard mercury/mercurous sulfate electrode (MSE) $\left[\mathrm{Hg} / \mathrm{Hg} \mathrm{SO}_{4}, \mathrm{~K}_{2} \mathrm{SO}_{4}\right.$ (saturated; +0.64 V vs standard hydrogen electrode, SHE)] from BASi, West Lafayette, IN, U.S.A. or a leakless silver/silver chloride electrode [Ag/AgCl, $1.0 \mathrm{M} \mathrm{KCl}$ from Cypress Systems, Lawrence, KS, U.S.A. (measured as $-0.006 \mathrm{~V}$ vs saturated calomel electrode, SCE, $\left[\mathrm{Hg} / \mathrm{Hg}_{2} \mathrm{Cl}_{2}\right.$, $\mathrm{KCl}$ (saturated)], which is equivalent to $+0.235 \mathrm{~V}$ vs SHE). The counter electrode was a graphite rod of diameter $6 \mathrm{~mm}$ from Sigma-Aldrich, Dorset, U.K. or a platinum foil from Goodfellow, Cambridge, U.K. All solutions were thoroughly saturated with hydrogen prior to both electrochemical experiments. All measurements were made under thermostated conditions at $25.0 \pm$ $0.5^{\circ} \mathrm{C}$.

\subsubsection{Ensemble and Nano-Impact Experiments}

All ensemble and nano-impact electrochemical experiments were performed on a three electrode system in a Faraday cage. The working electrode was a gold microdisc electrode of diameter 10.0 $\mu \mathrm{m}$ from ALS, Tokyo, Japan, and the electrode was polished with alumina powders from Buehler, Lake Bluff, IL, U.S.A., of decreasing sizes: 1.0, 0.3, and $0.05 \mu \mathrm{m}$. A leakless silver/silver chloride 
electrode [Ag/AgCl, 1.0 M KCl] from Cypress Systems, Lawrence, KS, U.S.A., functioned as a reference electrode (measured as $-0.006 \mathrm{~V}$ vs saturated calomel electrode, $\mathrm{SCE},\left[\mathrm{Hg} / \mathrm{Hg}_{2} \mathrm{Cl}_{2}, \mathrm{KCl}\right.$ (saturated)], which is equivalent to $+0.235 \mathrm{~V}$ vs SHE). A platinum foil from Goodfellow, Cambridge, U.K., acted as a counter electrode. All experiments were conducted under a hydrogen atmosphere. All electrochemical measurements were thermostated at $25 \pm 0.5^{\circ} \mathrm{C}$.

Potential and impact current measurements were controlled by an in-house built low noise, highly stabilized (1 kHz bandwidth) classic adder potentiostat, as previously described. ${ }^{35}$ An NI USB-6003 data acquisition (DAQ) device from National Instruments, Austin, TX, U.S.A was connected to a computer interface via an USB. Python 3.5 (Canopy from Enthought, Austin, TX, U.S.A) was employed to control the DAQ. The working electrode was running to ground where a low-noise current amplifier LCA-4K-1G from FEMTO, Messtechnik GmbH, Germany was used to measure currents. The DAQ device oversampled and digitized the incoming analogue signal at a stream rate of $100 \mathrm{kHz}$, the digitised signal was subsequently filtered at a bandwidth of $100 \mathrm{~Hz}$.

\subsection{Characterization}

The stock mesoporous and solid PtNP samples were characterized via transmission electron microscopy (TEM) and ultraviolet-visible (UV/vis) spectroscopy.

\subsubsection{TEM}

TEM (JEOL JEM-3000F FEGTEM, $300 \mathrm{kV}$ accelerating voltage or JEOL JEM-2100, $200 \mathrm{kV}$ accelerating voltage) was performed using conventional bright field imaging to determine the size of mesoporous PtNPs. Samples were prepared by depositing a drop of the stock mesoporous or solid PtNP suspension, with concentration $3.3 \times 10^{13}$ particles $\mathrm{L}^{-1}$ or $100 \mathrm{mg} \mathrm{L}^{-1}$ onto a carbon TEM grid coated with holey carbon film from Agar Scientific, Stansted, U.K. Air drying was allowed before any further examination. ImageJ software ${ }^{36}$ developed at the National Institutes of Health, U.S.A., was used to analyze the TEM images. Clearly defined mesoporous PtNPs consisting of an aggregation small particles or solid PtNPs were measured and both radii calculated. Example images are shown in SI. A total count of 86 mesoporous PtNPs gave a mean radius of $23.1 \pm 2.1 \mathrm{~nm}$, close to the value of $25 \mathrm{~nm}$ provided by the manufacturer; 49 small particles were averaged to give a mean radius of $2.0 \pm 0.3 \mathrm{~nm}$. To clarify, in this paper, the "mesoporous PtNP" refers to the mesoporous $23.1 \mathrm{~nm}$ particle, and the "small particles" are components of a mesoporous PtNP. A total count of 118 solid PtNPs gave a mean radius of $26.7 \pm 9.5 \mathrm{~nm}$, close to the value of $25 \mathrm{~nm}$ provided by the manufacturer. 


\subsubsection{UV/Vis Spectroscopy}

For UV/vis (Shimadzu UV-1800 UV/vis spectrophotometer) measurements, the stock mesoporous or solid PtNP suspension was diluted by a factor of 2 with ultrapure water to obtain absorbance in an analytically meaningful range. Both deuterium and halogen light sources were used, with the sample being scanned from 900 to 200 or $236 \mathrm{~nm}$. A broad surface plasmon peak around 295 or 285 $\mathrm{nm}$ was recorded indicating the presence of mesoporous or solid PtNPs. ${ }^{37}$

\section{Results and Discussion}

This work commences by outlining the behaviour of individual mesoporous platinum nanoparticles, a macroscopic platinum electrode and random arrays of platinum nanoparticles towards the oxidation of hydrogen. Analysis methods enabling the rigorous characterisation of the single particle response are employed, enabling direct comparisons to be drawn between the hydrogen oxidation behaviour of the differing platinum structures (macro, micro array and nanoparticulate). Under high mass-transport conditions (ca. $0.15 \mathrm{~m} \mathrm{~s}^{-1}$ ) the oxidation of hydrogen exhibits two voltammetric peaks at high overpotentials, in the region where the rate of reaction is limited by the adsorption kinetics of hydrogen to the catalytic interface. Through the study of the dependency of the platinum response - under high mass-transport conditions - to the solution phase $\mathrm{pH}$, the sensitivity of the adsorption rate to the interfacial platinum surface structure is demonstrated. Finally, the article turns to consider the time-current response of individual nanoparticles; highlighting, first, the contribution of under potential deposited hydrogen towards the oxidative current at the short timescale and second, through comparison with the response of particles of a different morphology (solid versus mesoporous), the catalytic activity and contribution of the internal structure is evidenced.

\subsection{Hydrogen Oxidation at Individual Nanoparticles}

The nanoparticles predominantly used in this article are $23.1 \pm 2.1 \mathrm{~nm}$ in radius and each particle is formed from an aggregate of smaller particles with radii $2.0 \pm 0.3 \mathrm{~nm}$ this leads to the particles having a mesoporous structure. In the final section of this article the individual nanoparticle response will be contrasted with that of a sample of solid particles. A representative TEM image of the mesoporous particles is shown in Figure $1 \mathrm{a}$ ), further sizing information is provided in the SI Section 1. As can be seen the particles have an open structure and have a relatively large surface area; assuming the constituent $2.0 \mathrm{~nm}$ particles form a closed packed structure, then the nanoparticles surface area can be calculated as being $~ 8.5$ times greater than that of the equivalent solid circumscribed sphere. 
First, a potentiostated gold microelectrode was immersed into a colloidal sol of mesoporous nanoparticles $(20 \mathrm{pM})$, saturated with hydrogen $(0.73 \mathrm{mM})$ and containing $20 \mathrm{mM} \mathrm{KNO}$. At potentials positive of $\sim-0.4 \mathrm{~V}$ vs $\mathrm{Ag} / \mathrm{AgCl}(1 \mathrm{M} \mathrm{KCl})$, small steps were observed in the measured current, an example chronoamperogram recorded at $+0.2 \mathrm{~V}$ is depicted in Figure $1 \mathrm{~b}$ ). These small steps in current are associated with the arrival of individual platinum catalytic entities to the electrode surface. Upon impact and electrical contact of the particles to the electrode surface, the HOR is electrochemically driven to occur at the newly arrived nanoparticulate material, and this leads to a measurable increase in the current. These small (pA) steps in current are superimposed upon a monotonically changing background associated with the capacitative charging of the gold electrode substrate. Notably, within the available electrochemical window and in the absence of nanoparticles, no 'steps' in current are observed; moreover, the gold electrode substrate itself is unable to catalyse the oxidation of hydrogen to any appreciable extent. Each new step represents the arrival of more new material to the interface and the steady increase in the catalytic current over the course of the reaction strongly indicates that the nanoparticles are irreversibly adsorbing to the electrode surface. In this experiment, only $20 \mathrm{mM} \mathrm{KNO}_{3}$ electrolyte is used to ensure the agglomeration of the nanoparticles in solution is minimised (see SI Section 2 for the associated UV/Vis spectra evidencing the meta stability of the sol in this millimolar ionic strength solution).
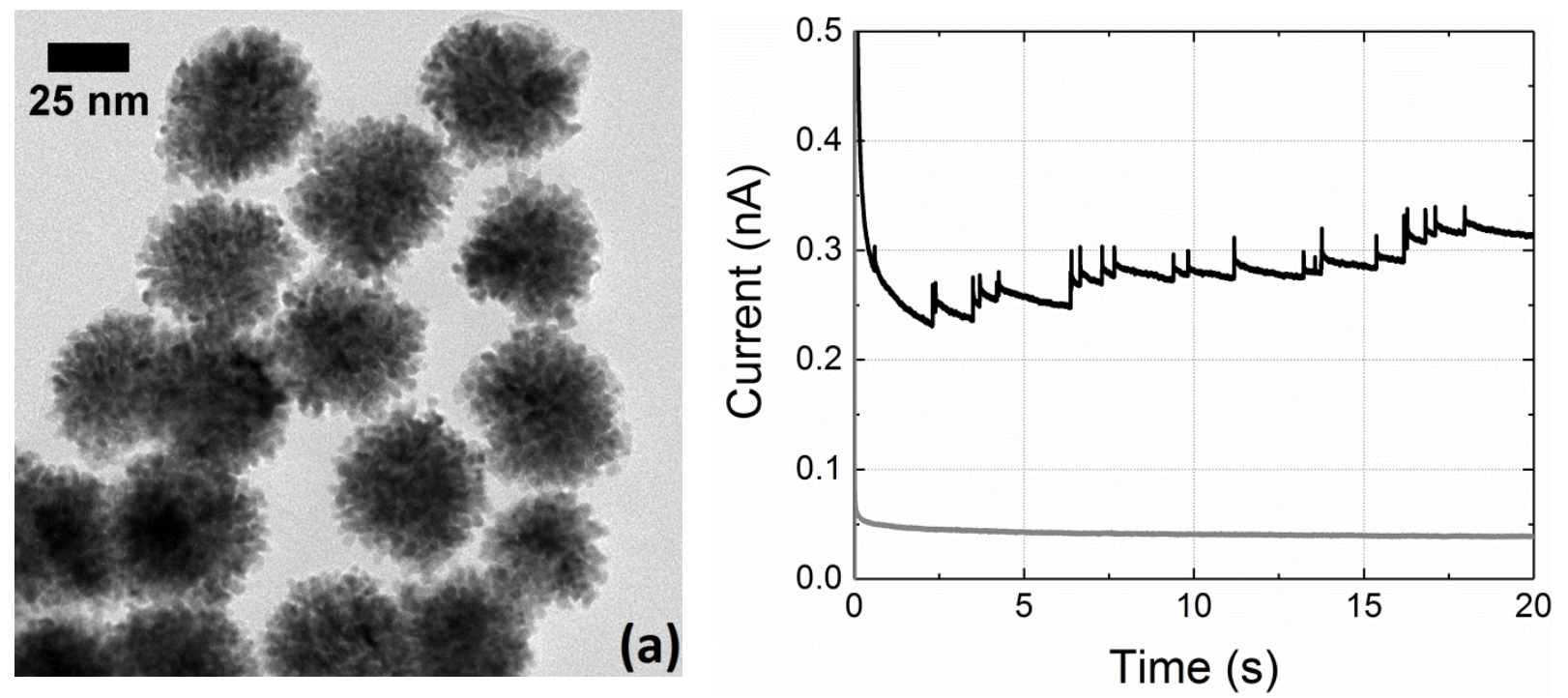

Figure 1 (a) TEM image of the mesoporous PtNPs. (b) Chronoamperogram of 20 pM mesoporous PtNPs in $20 \mathrm{mM} \mathrm{KNO} 3$ at a gold microelectrode with an applied potential of $0.2 \mathrm{~V}$ (black line), the average step in current is $11.1 \mathrm{pA}$, as measured $200 \mathrm{~ms}$ after the individual feature onsets; overlaid is a chronoamperogram in absence of impacting nanoparticles (grey line).

The arrival of a nanoparticle at the gold surface and the corresponding recorded steps 'up' in current, yield a direct measure of the rate of reaction at an individual nano entity. These steps in current, examples of which are shown in Figure 1 a), are only a few tens of picoamperes (11.1 pA on 
average, $200 \mathrm{~ms}$ after the onset of the event and at a potential of $+0.2 \mathrm{~V}$ vs $\mathrm{Ag} / \mathrm{AgCl}$ in $1 \mathrm{M} \mathrm{KCl})$ in magnitude and, as will be discussed below, the steps vary both in their shape and height as a function of potential but also vary between each stochastic event. First, however it is possible to calculate the expected current per particle if the reaction was limited by the rate of mass-transport of hydrogen to the nanoparticle. For a sphere on a flat surface the diffusional mass-transport limit to the particle is equal to $4 \ln (2) \pi n F D C r_{\mathrm{np}},{ }^{38}$ where $r_{\mathrm{np}}$ is the radius of the nanoparticle and is the bulk concentration of hydrogen. From this the diffusional mass-transport limited flux to an isolated particle at the electrode surface is theoretically predicted to be $\sim 142 \mathrm{pA}$. Relatedly, the diffusional mass-transport coefficient for material to/from such an isolated single particle on an electrode surface is equal to $\ln (2) D / r$, and for the present case of hydrogen oxidation at a $23.1 \mathrm{~nm}$ particle this corresponds to a rate of $\sim 0.15 \mathrm{~m} \mathrm{~s}^{-1}$. Given the steps in current for the individual nanoparticles are only a few picoamperes in magnitude as compared to more than $100 \mathrm{pA}$, it is concluded that the nanoparticle response is limited at this single particle scale not by the mass-transport of hydrogen to the catalytic surface but due to the reaction being kinetically limited by the rate of the surface reaction. Consequently, the variability in the response of individual nanoparticles for a given set of conditions reflects the heterogeneity of the nanoparticle sample both in terms of the nanoparticle dimensions and also the individual particle's inherent activity towards catalysis of the HOR.

\subsection{HOR at a Macroelectrode and Random Nanoparticle Arrays}

To enable comparison of the nanocatalytic response to be made with that of the bulk material the oxidation of hydrogen was studied at a polycrystalline Pt macroelectrode (radius $=0.83 \mathrm{~mm}$ ), the response of which is shown in Figure 2 a) blue line. Under these low mass-transport conditions (ca. $5 \times 10^{-4} \mathrm{~m} \mathrm{~s}^{-1}$ ) the oxidation of hydrogen at the macroelectrode is fully reversible with an associated mid-point potential of $-0.42 \mathrm{~V}$ vs $\mathrm{Ag} / \mathrm{AgCl}$ in $1 \mathrm{M} \mathrm{KCl}$ (i.e. $-0.185 \mathrm{~V}$ vs SHE); this is in excellent agreement with the thermodynamically predicted value of $-0.43 \mathrm{~V}$, the calculation of which is outlined in the SI section 4. It should be commented that although the present experiment is not performed under standard conditions and hence the oxidation does not occur at $0.0 \mathrm{~V}$ vs SHE, the redox potential is still thermodynamically well-defined in this system. The SI section 4 provides further information on the calculation of the characteristic oxidation potential for hydrogen under the non-buffered experimental conditions. The use of non-buffered conditions is important in relation to the latter half of the paper in which experimental work is undertaken in the absence of added electrolyte. Finally, also observable in the voltammetric response of the macroelectrode, as shown in Figure 2 a) blue line, is the underpotential deposition of hydrogen $\left(\mathrm{H}_{\text {upd }}\right)$ signal at $\sim-0.2 \mathrm{~V}$ that is situated in the diffusional tail of the hydrogen oxidation response. 
Due to the apparently fully irreversible accumulation of the platinum nanoparticles at the gold microelectrode surface (as evidenced in Section 3.1) the electrochemical response of a random array of adsorbed nanoparticles can be readily experimentally measured. For a gold electrode immersed in a platinum nanoparticle suspension, the cyclic voltammetric response of the electrode changes as a function of the electrode immersion time. The bare gold surface is unable to catalyse the HOR; consequently, due to the adsorption of the nanoparticles on to the gold interface and by the particles providing an increasingly large surface available to catalyse the HOR, the cyclic voltamemtric current increases as a function of the time the electrode is exposed to the nanoparticle suspension. SI section 5 provides example data showing the increase of the hydrogen oxidation response of a gold microelectrode as individual nanoparticle become attached to the electrode surface. Figure 2 a) (red line) depicts a voltammogram of a gold microelectrode with mesoporous nanoparticles adsorbed to the interface, where the electrode has been in the suspension for $\sim 5$ mins. Here, a welldefined wave corresponding to the catalytic oxidation of hydrogen at the nanoparticle array is observed and is seen to onset at the same potential as found for the platinum macroelectrode.
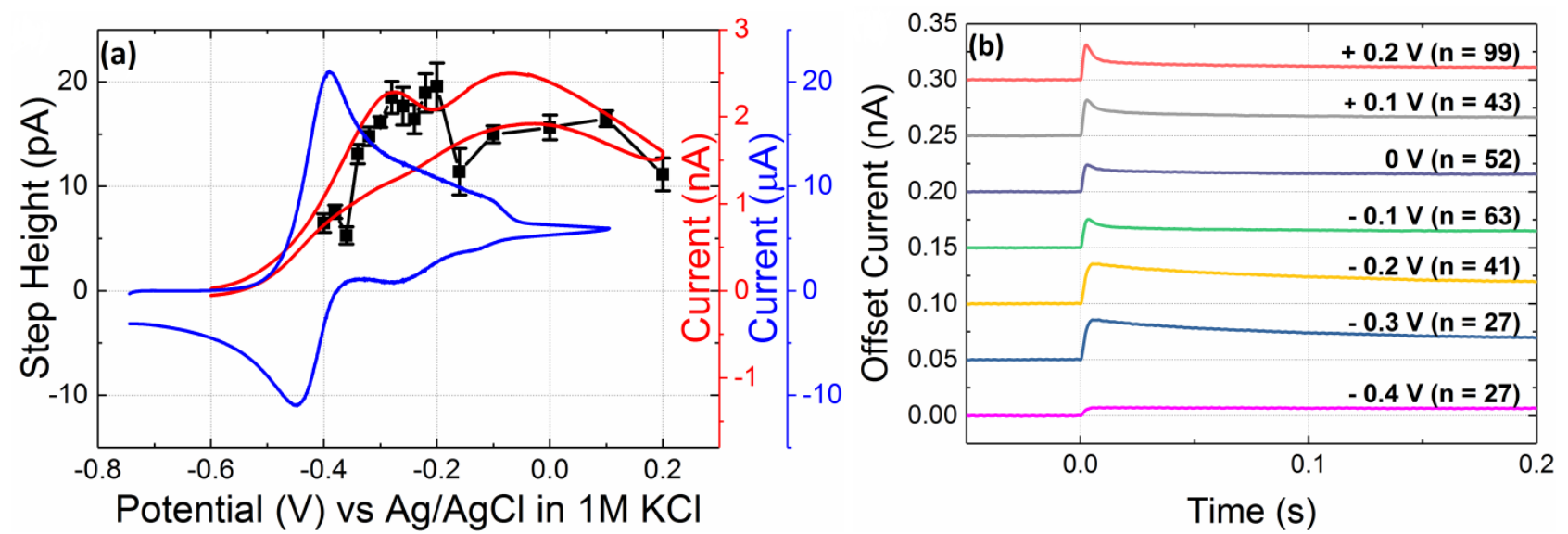

Figure 2. (a) The cyclic voltammograms for HOR on a Pt macro-electrode (blue; CV of scan rate $100 \mathrm{mV} \mathrm{s}^{-1}$, electrode of radius $831.5 \mu \mathrm{m}$ ) and an Au microelectrode with a random array of $\sim 140$ adsorbed platinum nanoparticles (red; CV of scan rate $200 \mathrm{mV} \mathrm{s}^{-1}$, nanoparticle of radius $23.1 \mathrm{~nm}$, electrode of radius $5 \mu \mathrm{m}, \sim 5 \mathrm{~min}$ nanoparticle accumulation time). The electrolyte is $0.2 \mathrm{M} \mathrm{KNO}_{3}$ for the Pt macroelectrodes and $20 \mathrm{mM}$ for the Au microlectrode, both solutions are saturated with $\mathrm{H}_{2}$. Overlaid is the variation in the magnitude of the current response of individual platinum nanoparticles as a function of the applied potential (black squares: measured from the time current transients, $200 \mathrm{~ms}$ after the nanoparticle arrival). (b) The averaged HOR response of individual platinum nanoparticles upon arrival - and electrical contact with - a potentiostated Au microelectrode as a function of the applied potential (vs $\mathrm{Ag} / \mathrm{AgCl}$ in $1 \mathrm{M} \mathrm{KCl}$ ). Current responses given as a function of time where $t=0$ is taken as the nanoparticle arrival and is associated with the step in current (the number (n) of individual features recorded at each potential is reported in the image). 
For this random array of platinum nanoparticles at the gold microelectrode an important consideration is the maximum current attainable across the entire nanoparticle array. In the limit of large numbers of particles accumulating at the interface then the nanoparticle array will comprise of enough catalytic sites that the HOR will hypothetically become limited, not by the surface reaction, but by the mass-transport of the hydrogen to the entire array. In this limit the current will be sensitive to the geometry and size of the substrate electrode. The diffusional mass-transport limited flux to the entire gold microelectrode (radius $=5 \mu \mathrm{m}$ ) is calculated to be $14.1 \mathrm{nA}$. For the data shown in Figure 2 a) at high overpotentials $(>-0.3 \mathrm{~V}$ ) the current is approximately $2.5 \mathrm{nA}$; consequently, this current is less than $20 \%$ of the maximum net current. Hence, to a reasonable approximation, the cyclic voltammetry of the nanoparticle array can be considered simply as a linear addition of the response of the individual nanoparticles i.e. the current per particle is taken to be effectively independent of the nanoparticle surface coverage. From the nano-impact data at +0.2 $\mathrm{V}$ the average step size of an individual event (Section 3.1) is found to have a magnitude of $11.1 \mathrm{pA}$, similarly at $+0.2 \mathrm{~V}$ the cyclic voltammetric response of the nanoparticle array, as depicted in Figure 2 a) has a current of $1.59 \mathrm{nA}$. Hence, the number of nanoparticles adsorbed on the gold electrode after five minutes of accumulation can be estimated for this voltammogram to be $\sim 140$.

The cyclic voltammetric response of the nanoparticle array evidences the presence of two peaks (at -0.3 and $-0.1 \mathrm{~V}$ ) in the electrochemical response at anodic potentials. These peaks are directly associated with the change in the catalytic activity of the nanoplatinum surface as a function of the applied potential. The physical origin of these peaks at higher over potentials will be returned to later in the text.

\subsection{Quantifying the Single Nanoparticle Behaviour}

The single nanoparticle towards the oxidation of hydrogen, as measured via chronoamperometry (cf. section 3.1), was investigated as a function of the applied electrode potential. To aid analysis of the nanoparticle impact results the average nanoparticle response for a given set of conditions is calculated (further details on this analysis procedure are provided in SI section 6). Figure 2 b) depicts the averaged response of individual particles from a sample of mesoporous platinum nanoparticles $\left(\mathrm{r}_{\mathrm{np}}=23.1 \mathrm{~nm}\right)$ towards the oxidation of hydrogen at a range of applied electrode potentials, each averaged current transient has been offset by 50 pA for clarity. Also shown in SI section 7 is the variation of the average nano-impact response at a potential of $+0.2 \mathrm{~V}$ to a variation of the solution phase hydrogen concentration, evidencing the change in the magnitude of the single particle response but not the shape. As can be seen from Figure 2 b) for all potentials more anodic than $-0.4 \mathrm{~V}$, the arrival of the nanoparticle to the electrode surface results in an abrupt increase in the catalytic current. Moreover, for all potentials above (and including) $-0.3 \mathrm{~V}$ a peak in the step 
response of the individual particles is observed at short times, the duration of this peak feature varies from $121 \mathrm{~ms}$ at $-0.3 \mathrm{~V}$ to $49 \mathrm{~ms}$ at $+0.2 \mathrm{~V}$ (as measured as a 90-10\% drop, taking the current at $200 \mathrm{~ms}$ after the spike onset as a reference). Furthermore, both the magnitude (in terms of charge) of the peak and the current in the long time limit (ca. $200 \mathrm{~ms}$ after the recorded particle arrival) also vary as a function of the applied potential.

The variation in the single nanoparticle step size as measured $200 \mathrm{~ms}$ after the onset of the nano-impact feature has been quantified and is shown in Figure 2 a) (black squares). The long time (200 ms) current response of the individual nanoparticles closely mirrors the response measured using cyclic voltammetry for the platinum nanoparticle array; the single nanoparticle response towards the oxidation of hydrogen also shows two peaks in the activity of the platinum nanomaterial at -0.1 and $-0.3 \mathrm{~V}$ vs $\mathrm{Ag} / \mathrm{AgCl}(1 \mathrm{M} \mathrm{KCl})$. These peaks, as will be outlined below, cannot be readily accounted for using the conventionally applied Tafel-Volmer reaction mechanism.

\subsection{Oxidative Mechanism: Tafel-Volmer}

At high-overpotentials (i.e. at potentials anodic of $-0.3 \mathrm{~V}$ vs $\mathrm{Ag} / \mathrm{AgCl}$ in $1 \mathrm{M} \mathrm{KCl}$ ), if the reaction rate at both the individual nanoparticles and the nanoparticle arrays are not limited by the masstransport of the hydrogen to the catalytic surface, what is the rate determining step? The relative invariance of the oxidation rate as a function of mass transport in this regime has conventionally been understood in terms of relating to the rate determining chemisorption of hydrogen to the electrode surface. ${ }^{14}$ The Tafel-Volmer reaction mechanism is commonly employed to describe the kinetics of the hydrogen oxidation reaction $(\mathrm{HOR})^{39}$ and is given by:

$\begin{array}{ll}\mathrm{H}_{2}+2 \mathrm{M} \rightleftharpoons 2 \mathrm{H}_{\mathrm{ads}} & k_{\mathrm{a}}, k_{\mathrm{d}} \\ \mathrm{H}_{\mathrm{ads}} \rightleftharpoons \mathrm{H}^{+}+e^{-}+\mathrm{M} & k_{\mathrm{el}}\end{array}$

where $\mathrm{M}$ and $\mathrm{H}_{\mathrm{ads}}$ represents an unoccupied and occupied surface adsorption sites respectively. The rate constants of hydrogen $\left(\mathrm{H}_{2}\right)$ adsorption and desorption are given by $k_{\mathrm{a}}\left(\mathrm{m}^{5} \mathrm{~mol}^{-2} \mathrm{~s}^{-1}\right)$ and $k_{\mathrm{d}}\left(\mathrm{m}^{2}\right.$ $\mathrm{mol}^{-1} \mathrm{~s}^{-1}$ ) respectively. The definition of the kinetic rate constants can be found in SI section 8 . The rate of electron transfer is commonly assumed to be well described using the Butler-Volmer formalism and hence the rate is defined by a rate constant $k_{\mathrm{el}}\left(\mathrm{m}^{3} \mathrm{~mol}^{-1} \mathrm{~s}^{-1}\right)$. It is on the basis of the involvement of an electron transfer in the Tafel reaction (and the similarly related Heyrovsky reaction) that it is concluded that at higher overpotentials, this process is not the rate determining step - were it to be controlling the electrochemical oxidation rate should exhibit a strong (exponential) dependency on the applied potential.

Through application of the Tafel-Volmer mechanism and solving the diffusion equation for the systems geometry, the expected voltammetric response for an array of diffusionally isolated 
spherical nanoparticles supported on an inert electrode surface can be simulated. The results of which are presented in Figure 3 a), where the simulated and experimental results $\left(20 \mathrm{mM} \mathrm{KNO}_{3}\right)$ have been overlaid. Full information on this simulation is provided in the SI section 8. For this mechanism the rate of electron transfer $\left(k_{\mathrm{el}}\right)$ the adsorption $\left(k_{\mathrm{a}}\right)$ and desorption $\left(k_{\mathrm{d}}\right)$ rate constants are unknowns. However, the magnitude of the adsorption rate constant $\left(k_{\mathrm{a}}\right)$ can be estimated from the individual nanoparticle impacts at high overpotential to be $4.3 \times 10^{7} \mathrm{~m}^{5} \mathrm{~mol}^{-2} \mathrm{~s}^{-1}$. Under conditions where the rate of reaction is limited by the chemi-sorption of hydrogen then the electrochemical oxidative flux (j) reduces to the simplified expression:

$j=2 k_{\mathrm{a}} c_{\mathrm{H}_{2}} \Gamma_{\mathrm{M}}^{2}$

where $c_{\mathrm{H}_{2}}$ is the surface concentration $\left(\mathrm{mol} \mathrm{m}^{-3}\right)$ of hydrogen and $\Gamma_{\mathrm{M}}$ is the surface coverage (mol $\mathrm{m}^{-2}$ ) of free active sites on the electrode surface. In the present simulation the values of $k_{\mathrm{d}}$ and $k_{\mathrm{el}}$ only serve to shift the position of the overall voltammetric wave.
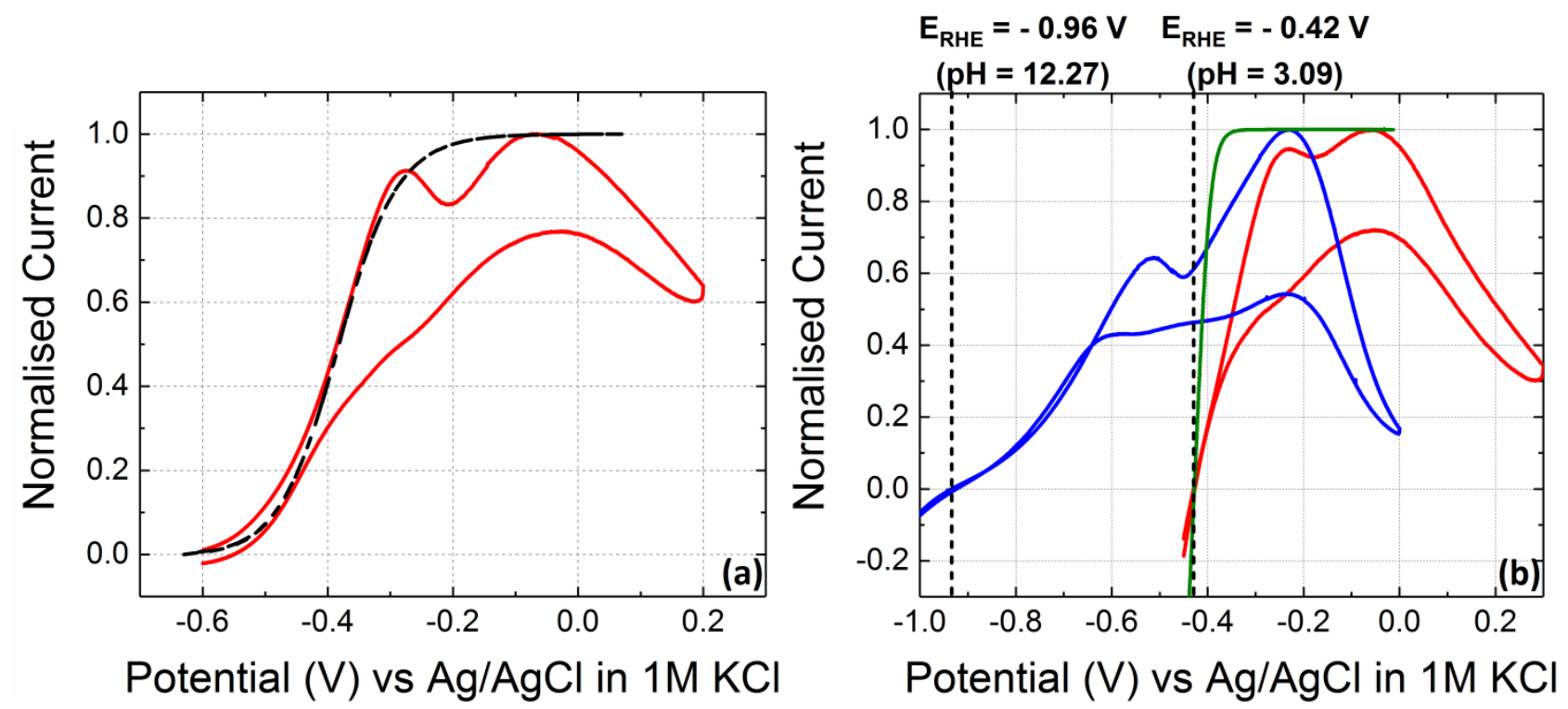

Figure 3. a) Depicts the simulated response for the oxidation of hydrogen via the Tafel-Volmer mechanism at an isolated spherical platinum nanoparticle under non-buffered conditions (equivalent to $20 \mathrm{mM} \mathrm{KNO}_{3}$ ) (dashed line). Kinetic parameters used in the simulation are $k_{\mathrm{a}}=4.3 \times 10^{7} \mathrm{~m}^{5} \mathrm{~mol}^{-2}$ $\mathrm{s}^{-1}, k_{\mathrm{d}}=4.3 \times 10^{7} \mathrm{~m}^{2} \mathrm{~mol}^{-1} \mathrm{~s}^{-1}$ and $k_{\mathrm{el}}=9.2 \times 10^{2} \mathrm{~m}^{3} \mathrm{~mol}^{-1} \mathrm{~s}^{-1}$. Overlaid for comparison is the experimental response ( $\mathrm{CV}$ of scan rate $200 \mathrm{mV} \mathrm{s}^{-1}$ ) of a platinum nanoparticle array at a gold microelectrode in $20 \mathrm{mM} \mathrm{KNO}_{3}$ (red line). b) Voltammetric response (CV of scan rate $200 \mathrm{mV} \mathrm{s}^{-1}$ ) of a gold microelectrode (radius $=5 \mu \mathrm{m}$ ) supporting a random array of platinum nanoparticles (radius $=23.1 \mathrm{~nm}$ ) under both acid (red: $1 \mathrm{mM} \mathrm{HClO}_{4}$ and $19 \mathrm{mM} \mathrm{KClO}_{4}$ ) and alkali conditions (blue: $20 \mathrm{mM} \mathrm{KOH}$ ), for clarity the calculated potentials for the RHE have been labelled for each $\mathrm{pH}$. Also shown is the theoretically predicted fully reversible response for a microelectrode towards the oxidation of hydrogen in the presence of $1 \mathrm{mM}$ protons (green line). 
As can be seen from Figure 3 a), although the Tafel-Volmer mechanism is capable of giving an accurate prediction of the experimental current at low overpotentials (cathodic of $-0.3 \mathrm{~V}$ ), this mechanism is unable to describe the voltammetric variation in the current at higher overpotentials in the 'adsorption limited regime'. For both the single particle impacts and the voltammetric response of the array of nanoparticles at the gold electrode, so-called 'fine-structure' is observed in the hydrogen oxidation signal as shown in Figure 2 a). This fine structure in $20 \mathrm{mM} \mathrm{KNO}_{3} \mathrm{Comprises}$ of two peaks in the reaction limited current situated at $\sim-0.3$ and $\sim-0.1 \mathrm{~V}$ vs Ag/AgCl in $1 \mathrm{M} \mathrm{KCl}$. The magnitude of these peaks in the reaction limited current and the fact that they are observable from analysis of the steady-state response (as measured after $200 \mathrm{~ms}$ ) of the individual nanoparticle means that these peaks are not directly relate to the oxidation of adsorbed hydrogen (i.e. $\mathrm{H}_{\text {upd); }}$; the feature is not simply related to the superposition of a surface process with the catalytic oxidation of hydrogen. Consequently, it is concluded that this variation in the oxidation rate in the adsorption limited regime must reflect a sensitivity and change in the adsorption rate constant, $k_{\mathrm{a}}$, or the surface coverage of the active sites on the catalytic interface, $\Gamma_{\mathrm{M}}$, with the applied potential. For the peak -0.1 V the peak likely arises from anion specific adsorption leading to a decrease in the surface coverage of active sites at high overpotentials. ${ }^{40}$ However, it is the origin of the peak at $-0.3 \mathrm{~V}$ that is the focus of the following section.

\subsection{Oxidative Mechanism: Behaviour at Variable pH}

The oxidation of hydrogen at the mesoporous nanoparticles was further studied under both alkaline and acidic conditions. A gold micro-electrode was immersed into a platinum nanoparticle sol containing either $20 \mathrm{mM} \mathrm{KOH}$ or $1 \mathrm{mM} \mathrm{HClO}_{4}$ and $19 \mathrm{mM} \mathrm{KClO}_{4}$. Under acidic conditions the nanoparticle suspension is found to be far less stable as has been recently reported in the literature, ${ }^{30}$ hence the use of concentration of only $1 \mathrm{mM} \mathrm{HClO}_{4}$ under acid conditions. Under both buffering conditions steps in the chronoamperometric response are observed corresponding to the arrival of individual particles to the electrode surface. However, although the rate of hydrogen oxidation under acidic conditions is comparable to the situation found for the individual nanoparticle response in $20 \mathrm{mM} \mathrm{KNO}_{3}$, the rate of hydrogen adsorption is found to be almost an order of magnitude lower under alkaline conditions (SI section 9).

Figure $3 \mathrm{~b}$ ) depicts the voltammetric response of a platinum nanoparticle array at a gold

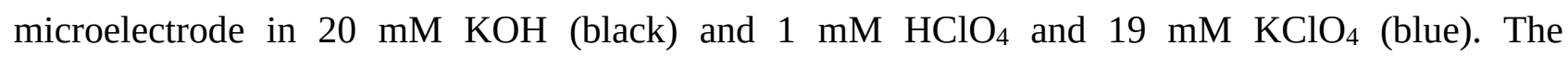
nanoparticle arrays were formed, as before, by allowing nanoparticles in the solution phase to randomly adsorb to the gold interface. The voltammograms were recorded after at least 2 minutes of electrode immersion. As evidenced by the reductive current at cathodic potentials, both 
voltammograms presented in $3 \mathrm{~b}$ ) show significant hydrogen evolution in the voltammetric window of study, this is in contrast to the non-buffered case presented above, where the lack of $\mathrm{pH}$ buffering inhibits this process from occurring. Figure $3 \mathrm{~b}$ ) demonstrates that the position of the voltammetric wave, as defined by the potential at which it crosses the x-axis, shifts in a Nernstian manner with $\mathrm{pH}$, as indicated by the calculated potential of the reversible hydrogen electrode (RHE), Figure $3 \mathrm{~b}$ ) dotted vertical lines. Moreover, the electron transfer kinetics are appreciably faster under acidic as compared to alkaline conditions, as evidenced by the voltammetric wave shape. However, although faster the voltammetric response under acid ( $\mathrm{pH}$ 3.1) conditions is not at the reversible limit, as can be seen from deviation of the response away from that analytically predicted for a Nernstian reaction occurring at a diffusionally isolated particle (derivation of the reversible Nernstian response is given in the SI section 10).

Figure 3b) also evidences that, although the $\mathrm{pH}$ has been altered dramatically ( 9.2 $\mathrm{pH}$ units), the minimum in the adsorption limited regime has only shifted a comparatively small amount. At $\mathrm{pH}$ 3.1 the local minimum in the reaction limited current occurs at $-0.18 \mathrm{~V}$, however at $\mathrm{pH} 12.3$ this has only been shifted by approximately $0.27 \mathrm{~V}$ to $-0.45 \mathrm{~V}$. As demonstrated in the SI section 11 , the $\mathrm{H}_{\text {upd }}$ signal for the platinum surface occurs at $\sim-0.2 \mathrm{~V}$ vs $\mathrm{Ag} / \mathrm{AgCl}$ in $1 \mathrm{M} \mathrm{KCl}$ at $\mathrm{pH} 3.1\left(1 \mathrm{mM} \mathrm{HClO}_{4}\right.$ and $19 \mathrm{mM} \mathrm{KClO}_{4}$ ) and at $-0.45 \mathrm{~V}$ at $\mathrm{pH} 12.3(20 \mathrm{mM} \mathrm{KOH}$, in the presence of citrate). Although the $\mathrm{H}_{\text {upd }}$ voltammetric signal is associated with the stoichiometric transfer of one proton per electron the position of the wave is known to be sensitive to specific anion adsorption and for polycrystalline platinum and for a number of crystal facets (most notably Pt 100 and Pt 110) the position of the wave varies with $40-50 \mathrm{mV} \mathrm{PH}^{-1}$ at $\mathrm{pHs}$ above $3 .{ }^{41-42}$ Moreover, conventionally, the position of the $\mathrm{H}_{\text {upd }}$ signal relative to the RHE has been used as a descriptor of the rate of electron transfer as a function of $\mathrm{pH} .{ }^{41}$

One interpretation for the 'fine-structure' in the activity of the hydrogen oxidation reaction at higher overpotentials previously provided in the recent literature, is that these two peaks in the adsorption limited regime correspond to two different adsorption sites and that at higher overpotentials both sites become blocked, plausibly due to competitive anion adsorption. ${ }^{15}$ Alternatively, we highlight that this local minimum in activity, as evidenced by the drop in catalytic current, occurs at potentials comparable to those at which the $\mathrm{H}_{\text {upd }}$ occurs on the underlying platinum surface. In the nonbuffered case (20 $\mathrm{mM} \mathrm{KNO}_{3}$ ) this can clearly be seen from Figure 2 a) where the local minima in the hydrogen adsorption rate occurs at potentials comparable to the $\mathrm{H}_{\text {upd }}$ signal as observed on the macroscopic platinum electrode. 
This conclusion that the particle activity initially decreases with the removal of $\mathrm{H}_{\text {upd }}$ from the surface is, at first sight, difficult to reconcile with the Tafel-Volmer mechanism. We can express the total surface coverage of the active sites as, $\Gamma_{\max }=\Gamma_{\mathrm{M}}+\Gamma_{\mathrm{H}(\mathrm{ads})}$, where $\Gamma_{\mathrm{H}(\mathrm{ads})}$ is the surface coverage of adsorbed hydrogen $\mathrm{H}(\mathrm{ads})$. The value of $\Gamma_{\max }$ is often assumed to be a constant and for a clean Pt electrode given the value of $\Gamma_{\max }=2.2 \times 10^{-5} \mathrm{~mol} \mathrm{~m}^{-2},{ }^{43}$ where it is assumed that $\mathrm{H}_{\text {upd }}$ is the same as $\mathrm{H}_{\text {ads. }}$. Whether $\mathrm{H}_{\text {upd }}$ represents the primary intermediate in the hydrogen oxidation reaction and is hence the same as $\mathrm{H}_{\mathrm{ads}}$ in the Tafel-Volmer mechanism is debatable. ${ }^{15}$ If $\mathrm{H}_{\mathrm{ads}}$ was the same as $\mathrm{H}_{\text {upd }}$ one would expect in accordance with the Tafel-Volmer mechanism that the oxidative removal of the $\mathrm{H}_{\text {upd }}$ would lead to an increase and not a decrease (as observed experimentally) in the surface reaction limited current. It is however, on the basis of the presented experimental data, reasonable to conclude that the first peak in the HOR adsorption limited region relates directly to a change in the interfacial structure as a function of the applied potential; as the surface coverage of adsorbed hydrogen $\left(\mathrm{H}_{\text {upd }}\right)$ decreases so too does the apparent hydrogen adsorption rate. Certainly due to lateral interaction between adsorbed hydrogens the enthalpy of adsorption would not be anticipated to be a constant as a function of the surface coverage of hydrogen. Ultimately we conclude that either the adsorption rate $\left(k_{\mathrm{a}}\right)$ or the number of available active sites must decrease at higher overpotential. This decrease in the catalytic rate reflects a changes in the interfacial structure either associated with the desorption of $\mathrm{H}_{\text {upd }}$ or due to specific anion adsorption.

\subsection{The Shape of Individual Nano-Impacts}

Having explored the operative mechanism, the article now returns to consider the shape of the 'step' response of individual nanoparticles, as exemplified in the data shown in Figure 2 b), and importantly explore the origin of the initial 'spike' in current at times less than $200 \mathrm{~ms}$ after the impact of a nanoparticle. This spike in current when integrated is experimentally found to vary in magnitude in the range of $0.1-1 \mathrm{pC}$. The onset of the spike in the single nanoparticle time-current transient correlates closely with the potential at which the $\mathrm{H}_{\text {upd }}$ signal is observed, this indicates that this process ( $\mathrm{H}_{\text {upd }}$ removal) provides at least a partial charge contribution to the observed spike in current. For the impact response of the platinum nanoparticles at $+0.2 \mathrm{~V}$, the integral of the charge under the initial spike gives a charge of $0.22 \mathrm{pC}$. However at $-0.3 \mathrm{~V}$ the charge associated with this initially non-steady state oxidation event is found to be $1.1 \mathrm{pC}$. Assuming the total nanoparticle surface area is covered in hydrogen then this charge is equivalent to surface coverages of $384 \mu \mathrm{C}$ $\mathrm{cm}^{-2}$ and $1.9 \mathrm{mC} \mathrm{cm}{ }^{-2}$, respectively. ${ }^{44}$ In contrast on polycrystalline platinum a monolayer of hydrogen has a surface coverage of $210 \mu \mathrm{C} \mathrm{cm}^{-2}$. Consequently, at high overpotential $(+0.2 \mathrm{~V}$ vs $\mathrm{Ag} / \mathrm{AgCl}$ in $1 \mathrm{M} \mathrm{KCl}$ ) it is reasonable to conclude that within the experimental uncertainty, the 
charge passed during this spike in current is associated simply with the removal of $\mathrm{H}_{\text {upd }}$ hydrogen from an individual platinum nanoparticle.

However, at lower potentials the far greater magnitude of the charge passed and its sensitivity to the applied potential indicates that at least one other process is occurring during the course of this spike in current. As highlighted in Sections 3.4 and 3.5 the interplay between $\mathrm{H}_{\text {upd }}$ surface coverage and the particle's activity towards the HOR is not simple. However, the 'excess' charge passed during the spike in current at low overpotentials most likely reflects the higher activity of the nanoparticle towards the oxidation of hydrogen at the initial onset of the reaction where the surface coverage of $\mathrm{H}_{\text {upd }}$ is higher. During the course of the spike in current, and as the surface coverage of $\mathrm{H}_{\text {upd }}$ decreases, the activity of the particle towards the catalytic oxidation of hydrogen is concomitantly lowered. Hence, this result again evidences the important role of the surface coverage of the $\mathrm{H}_{\text {upd }}$ on the platinum surface and that the rate of the hydrogen oxidation reaction is not simply directly controlled by the applied potential. In summary, the charge passed during the initial spike in current for an individual impacting nanoparticle at electrode potentials between -0.3-+0.1 V (vs. Ag/AgCl) - as shown in Figure 2 b) - is large than anticipated simply for the removal of a monoloayer of $\mathrm{H}_{\text {upd }}$ hydrogen for the platinum nanosurface. The extra charge is consistent with arising due to the activity of the particle towards the catalytic oxidation of hydrogen decreasing as $\mathrm{H}_{\text {upd }}$ hydrogen is removed from the particle's surface; the two processes are not independent.

In light of the above discussion, models of the single nanoparticle catalytic oxidation event are developed in which the transfer function and hence the bandwidth of the measurement electronics are also accounted for (further details are provided in the SI section 12). These models focus on describing the single particle current transients at $+0.2 \mathrm{~V}$ where the charge passed during the spike in current is consistent with arising predominantly from the removal of a monolayer of $\mathrm{H}_{\text {upd }}$ from the impacting particle surface. We further note that, upon arrival of the nanoparticle to the electrode surface and the formation of an electrical contact, the nanoparticle will become charged. This nanoparticle charging will lead to the occurrence of a small capacitative current. If we assume that the double layer capacitance associated with the platinum surface is $20 \mu \mathrm{F} \mathrm{cm}{ }^{-2}$ then if nanoparticle changes its potential by one volt then the maximum expected capacitative charge per individual nanoparticle charging event is only anticipated to be of the order of $0.01 \mathrm{pC}$, this value is at least an order of magnitude less than is found experimentally for the charge passed during the spike (cf. 0.2 pC). Consequently, in the following discussion only the Faradaic single nanoparticle response is considered. 
Upon application of a potential to a nanoparticle the timeframe for a reaction to reach the steadystate for a diffusionally limited process is given approximately by $r_{\mathrm{np}}{ }^{2} / D(\sim 125 \mathrm{~ns}$ in the present case), given the near reversible nature of HOR on platinum, other surface reaction processes may be anticipated to reach steady-state on a similarly rapid timescale; consequently, in the absence of other contributing factors a simple step in the current time profile should be anticipated to occur after the arrival of a single nanoparticle entity at the electrode surface. Figure 4 a) (red line) presents the expected current transient for a single impacting nanoparticle where the rate at which the measured current increases is determined by the bandwidth of the measurement electronics then, for the device used in this work the steady-state response should be reached within approximately 10 ms of the onset of the nanoparticle impact. As shown in the Figure 2 b), at cathodic potentials $<-0.3$ $\mathrm{V}$ (vs $\mathrm{Ag} / \mathrm{AgCl}$ in $1 \mathrm{M} \mathrm{KCl}, 20 \mathrm{mM} \mathrm{KNO}_{3}$ ) the single nanoparticle responses are found to be of a simple 'step' form and are well described by this simple catalytic reaction only model. But this first model is incapable of explaining the initial peak in the time current transients as observed experimentally at $+0.2 \mathrm{~V}$.

The second model, Figure 4 a) blue line, looks to account for the oxidation of surface adsorbed hydrogen $\left(\mathrm{H}_{\text {upd }}\right)$ at only the outer surface of the particle. The oxidation of the $\mathrm{H}_{\text {upd }}$ on the external particle surface is assumed to occur very rapidly, consequently, the resulting step shape is that of a superposition of a step and an impulse response as depicted in Figure 4 a) blue line. Here the current rises rapidly but the response of the system is not predicted to result in an appreciable peak at short times. Consequently, this clearly demonstrates that the internal structure of the mesoporous nanoparticle contributes to the peak observed experimentally for the individual nanoparticles.

The third model, Figure 4 a) green and yellow lines; the reaction limited current, the near instantaneous oxidation of $\mathrm{H}_{\text {upd }}$ on the external surface and a slower oxidation of the $\mathrm{H}_{\text {upd }}$ contained on the internal nanoparticle surface are all accounted for. Due to the mesoporous structure of the nanoparticle their surface area is approximately 8.5 times greater than that of a sphere of an equivalent (circumscribed) size. Moreover, to provide a simplified model of the oxidation of the internal $\mathrm{H}_{\text {upd }}$ it is assumed that the reaction is limited by the diffusion of the species out of the sphere. Consequently, the rate at which the internal hydrogen is oxidised is sensitive to an applied effective rate constant and the geometry of the system. The results of this model are shown in Figure 4 a) where the green and yellow lines have associated effective diffusion coefficients of $1 \times 10^{-14}$ and $1 \times 10^{-15} \mathrm{~m}^{2} \mathrm{~s}^{-1}$, respectively. This model successfully reflects the experimental shape of the nano-impact response and provides a clear parameterisation of the observed spike shape, evidencing the likely relatively slow mass-transport through the internal particle structure. The 
direct comparison between this rather simple model and the experimental data reveals an excellent qualitative agreement of theory and experiment. Owing to the simplicity of the theoretical model, there are though, notable difference between the modelled and the measured spike shapes. Primarily, the rate at which the current profile tends to steady-state and the magnitude of the peak response are both sensitive to the applied potential in the experimental work, potentially indicating the importance of migratory forces on material transport through the particle’s internal structure.
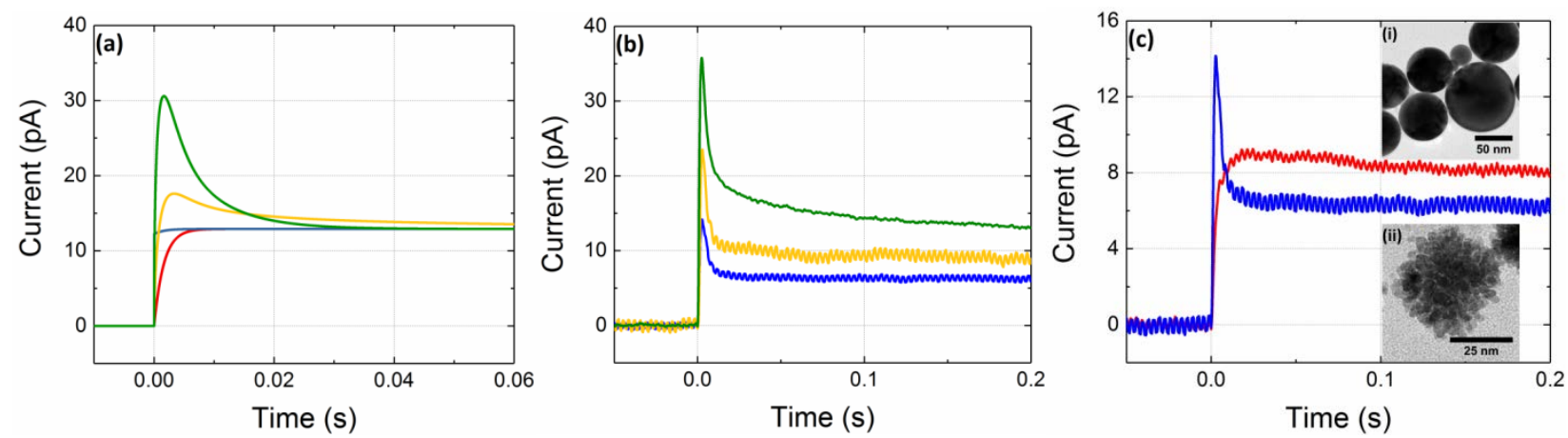

Figure 4. a) Theoretically predicted impact response for the oxidation of hydrogen at individual nanoparticles accounting for the transfer function of the measurement device: red line; reaction limited process only, blue line; reaction limited response plus the oxidation of hydrogen adsorbed on the external nanoparticle surface, yellow and green lines; reaction limited current and a contribution at short times from both the oxidation of hydrogen adsorbed on the external and internal surface where the mass transport through the particle has been modelled using an 'effective' diffusion coefficient (see text) of $1 \times 10^{-15}$ and $1 \times 10^{-14} \mathrm{~m}^{2} \mathrm{~s}^{-1}$ (yellow and green respectively) b) experimentally determined average response of mesoporous platinum nanoparticles towards the oxidation of hydrogen at $+0.2 \mathrm{~V}$ vs $\mathrm{Ag} / \mathrm{AgCl}(1 \mathrm{M} \mathrm{KCl})$ in the presence of $20 \mathrm{mM}, 2 \mathrm{mM}$ and $0 \mathrm{mM}$ (green, yellow and blue respectively) additional $\mathrm{KNO}_{3}$ electrolyte. (c) Averaged chronoamperograms for the oxidation of hydrogen with mesoporous (blue) and solid (red) PtNPs in the absence of additional electrolyte.

\subsection{Impacts in the Absence of Added Electrolyte and the Response of Solid Nanoparticles}

In the previous section for the provided theoretically models it was demonstrated that the initial spike in current at $+0.2 \mathrm{~V}$ is consistent with arising from the removal of $\mathrm{H}_{\text {upd }}$ from the nanoparticle's mesoporous surface. The magnitude of the initial spike is, on this basis, anticipated to be sensitive to the nanoparticle structure. This final section of the article looks to explore experimental evidence for this conclusion. This will be achieved first by investigating the sensitivity of the nano-impact response to the presence of added electrolyte. Second, the influence of changing the nanoparticle morphology on the response of a single particle will be reported. 
Figure $4 \mathrm{~b}$ ) depicts the average hydrogen oxidation transients for a series of individual particles studied with variable concentrations of added electrolyte. A gold microelectrode was immersed into a mesoporous platinum nanoparticle suspension, and the electrode potential held at $+0.2 \mathrm{~V}$ vs $\mathrm{Ag} / \mathrm{AgCl}$ in $1 \mathrm{M} \mathrm{KCl}$. The nanoparticle solution was saturated with hydrogen and had a variable concentration of added supporting electrolyte (20, 2 and $0 \mathrm{mM} \mathrm{KNO}_{3}$ ). The nanoparticle suspension used within this work contains citrate as a stabilising capping agent; consequently, even in the absence of additional electrolyte a solution phase concentration of $\sim 0.2 \mathrm{mM}$ citrate will be present in the nanoparticle suspension. As shown in Figure $4 \mathrm{~b}$ ), even in the absence of the addition of electrolyte, oxidative events associated with the arrival of platinum nanoparticles to the electrode surface are observed. Most notably the magnitude of the individual nanoparticle impact events decrease as a function of the electrolyte concentration; however, the spike shape is relatively conserved (SI section 13 depicts the normalised impact responses allowing direct comparison of the oxidative spike shapes).

If the reaction is limited by the rate of the surface reaction why is the magnitude of the single nanoparticle response (as evidence in Figure 4b) sensitive to the supporting electrolyte concentration? For the internal surfaces it is reasonable that the hydrogen oxidation reaction at this interface may be limited by a number of factors, including, the solution phase potential drop through the particle structure and/or the transport of either the reactant (hydrogen) or product (protons) in or out of the internal structure. Due to hydrogen being neutral the mass-transport of this material is not influenced to any appreciable extent by the local electric field; however, the product (protons) will be. It has previously been found that the oxidation of hydrogen at a platinum microelectrode can be studied under aqueous conditions in the absence of additional electrolyte, this occurs without significant distortion of the voltammetric response. ${ }^{34}$ For the mesoporous nanoparticles in the absence of additional electrolyte the solution phase concentration will be approximately $29 \mu \mathrm{S} \mathrm{cm}^{-1}$ as based upon the known experimental citrate concentration (0.24 mM) and its estimated molar conductivity of $120 \mathrm{~S} \mathrm{~cm}^{2} \mathrm{~mol}^{-1} \cdot{ }^{45}$ Hence, on the nanoscale very significant potential drops may occur through the solution phase of the internal nanoparticle structure. ${ }^{46}$ Consequently, it is concluded that the used electrolyte concentration serves to control the ohmic drop through the nanoparticle internal structure; under conditions of lower ionic strength the hydrogen oxidation reaction is likely restricted to occurring at a thinner layer near the external

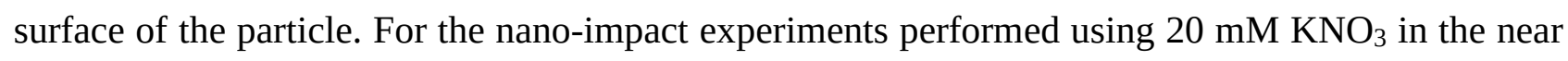
steady-state regime (>200 ms) the hydrogen oxidation reaction on the internal particle surface contributes at least $40 \%$ of the catalytic current of the individual particle. However, the primary conclusion of this result is that; at the nanoscale electrochemically reactions can be driven without 
the addition of excess supporting electrolyte. As will be discussed below this has important consequence for the range of materials that can be studied using this technique.

Figure 4 c) shows a comparison of an average spike event measured for the mesoporous nanoparticle and the same experiment performed using solid platinum nanoparticles (SI section 1 provides further TEM characterisation of these different materials) both recorded in the absence of additional electrolyte. Inlayed in Figure 4 c) are representative TEM images of the material showing the differing internal structure of the nanoparticles. These solid nanoparticles are formed via laser ablation and consequently have a relatively large size distribution moreover, the material is found to be comparatively highly sensitive to the addition of supporting electrolyte. Consequently, the impact response of these solid platinum nanoparticles cannot be readily studied in the presence of additional electrolyte due to their lack of stability towards agglomeration. However, the averaged impact response of the solid nanoparticles (Figure $4 \mathrm{c}$ )) as recorded in the absence of addition electrolyte does not exhibit a significant peak in the current transients at short times. Thus providing strong evidence, that the peak in the nanoparticle response of the individual mesoporous platinum nanoparticles, is directly related to the morphology of the nanostructure.

Both the alteration of the magnitude of the nanoparticle impact response as a function of the electrolyte concentration and the changed shape of the impact response between the mesoporous and solid particles serves to evidence the activity of the internal mesoporous nanoparticle structure towards the oxidation of hydrogen. The observation of the variability the HOR at high overpotentials such that the reaction rate decreases at high overpotentials, yields insight into the origin of the observed sensitivity of the magnitude of the transient spike as a function of potential; at lower overpotentials the internal structure is more active towards hydrogen oxidation and likely varies in activity not directly with the applied potential but is sensitive to the internal $\mathrm{H}_{\text {upd }}$ coverage, where equilibration of the internal $\mathrm{H}_{\text {upd }}$ surface coverage is slowed by the transport through the particles internal structure.

\section{Conclusions}

This work has demonstrated that even at the single particle scale the oxidation of hydrogen at platinum, the material's catalytic activity is sensitive to the applied potential and exhibits two maxima in activity. These peaks in activity are directly reflected in the electrochemical current measured at both individual particles and sparse arrays of material. In contrast to the literature the 
presence of the two peaks in activity are not ascribed as relating to the presence of two different catalytic sites but by studying this reaction under different proton concentrations the position of the first minima is shown to directly correlate with the potential of $\mathrm{H}_{\text {upd }}$ oxidation. The rate of adsorption of hydrogen to the interface is consequently highly sensitive to the interfacial structure, where at higher potentials the HOR is likely inhibited by specific anion adsorption. This conclusion is corroborated by the shape of the individual nanoparticle impact transients, where at low potentials the activity of the platinum particles dynamically decreases with time as $\mathrm{H}_{\text {upd }}$ is removed from the particle's interface.

Having provided greater insight into the hydrogen oxidation reaction at these high current densities the shape of the resulting single particle time-current transients also is shown to be sensitive to the structure of the nanomaterial. For the mesoporous particles used in this work the activity of the internal structure towards the oxidation of hydrogen is directly evidenced via comparison with solid particles.

\section{Supporting Information}

Electron microscopy and characterisation of the platinum nanoparticles, UV-vis experiments evidencing the colloid stability in millimolar electrolyte concentrations, examples of chronoamperograms showing the arrival and leaving of individual catalytic nanoparticles, the characteristic potential for hydrogen oxidation under non-standard conditions, accumulation of platinum nanoparticle at a gold microelectrode, procedure for averaging nano-impact step responses, variation of the mesoporous nanoparticle response as a function of hydrogen concentration, TafelVolmer mechanism at an isolated nanoparticle on a plane, mesoporous platinum nanoparticle impacts under acid and alkali conditions, Nernstian response of a microelectrode towards proton/hydrogen redox couple, $\mathrm{H}_{\text {upd }}$ on polycrystalline and nanoparticulate surfaces, computational modelling of current spike shapes, comparison of the average nanoparticle response as a function of electrolyte concentration.

\section{Acknowledgements}

The reseach is sponsored by the funding from the European Research Council under the European Union Seventh Framework Programme (FP/2007-2013)/ERC Grant Agreement No. [320403]. X.J. thanks the China Scholarship Council for supporting her DPhil research.

\section{References}

1. Snyder, J.; Fujita, T.; Chen, M.; Erlebacher, J., Oxygen reduction in nanoporous metal-ionic liquid composite electrocatalysts. Nat. Mater. 2010, 9, 904-907.

2. Marcus, R. A., On the theory of oxidation - reduction reactions involving electron transfer. I. J. Chem. Phys. 1956, 24, 966-978. 
3. Hush, N. S., Adiabatic theory of outer sphere electron-transfer reactions in solution. Trans. Faraday Soc. 1961, 57, 557-580.

4. Hush, N. S., Adiabatic rate processes at electrodes. I. Energy-charge relationships. J. Chem. Phys. 1958, 28, 962-972.

5. Chidsey, C. E., Free Energy and Temperature Dependence of Electron Transfer at the Metal/Electrolyte Interface. Science 1991, 251, 919-922.

6. Xiao, X.; Bard, A. J., Observing single nanoparticle collisions at an ultramicroelectrode by electrocatalytic amplification. J. Am. Chem. Soc. 2007, 129, 9610-9612.

7. Albrecht, T.; Horswell, S.; Allerston, L. K.; Rees, N. V.; Rodriguez, P., Electrochemical Processes at the Nanoscale. Curr. Opin. Electrochem. 2017.

8. Dickinson, E. J. F.; Compton, R. G., Influence of the diffuse double layer on steady-state voltammetry. J. Electroanal. Chem. 2011, 661, 198-212.

9. Murray, R. W., Nanoelectrochemistry: metal nanoparticles, nanoelectrodes, and nanopores. Chem. Rev. 2008, 108, 2688-2720.

10. Favaro, M.; Jeong, B.; Ross, P. N.; Yano, J.; Hussain, Z.; Liu, Z.; Crumlin, E. J., Unravelling the electrochemical double layer by direct probing of the solid/liquid interface. Nat. Commun. 2016, 7, 12695.

11. Sheng, W.; Gasteiger, H. A.; Shao-Horn, Y., Hydrogen oxidation and evolution reaction kinetics on platinum: acid vs alkaline electrolytes. J. Electrochem. Soc. 2010, 157, B1529-B1536.

12. Markovića, N. M.; Sarraf, S. T.; Gasteiger, H. A.; Ross, P. N., Hydrogen electrochemistry on platinum low-index single-crystal surfaces in alkaline solution. J. Chem. Soc., Faraday Trans. 1996, 92, 3719-3725.

13. Ledezma-Yanez, I.; Wallace, W. D. Z.; Sebastián-Pascual, P.; Climent, V.; Feliu, J. M.; Koper, M., Interfacial water reorganization as a $\mathrm{pH}$-dependent descriptor of the hydrogen evolution rate on platinum electrodes. Nat. Energy 2017, 2, 17031.

14. Frumkin, A.; Aikazyan, E., Kinetics of ionization of molecular hydrogen on platinum electrodes. Russ. Chem. Bull. 1959, 8, 188-197.

15. Zalitis, C.; Kucernak, A.; Sharman, J.; Wright, E., Design principles for platinum nanoparticles catalysing electrochemical hydrogen evolution and oxidation reactions: edges are much more active than facets. J. Mater. Chem. A 2017, 5, 23328-23338.

16. Kongkanand, A.; Mathias, M. F., The priority and challenge of high-power performance of low-platinum proton-exchange membrane fuel cells. J. Phys. Chem. Lett. 2016, 7, 1127-1137.

17. These high rates are attained through the use of a gas diffusion electrode ensuring a rapid supply of material to the interface. Assuming an anode loading of $0.05 \mathrm{mg} \mathrm{cm}^{-2}$ with a catalyst electrochemical surface area of $50 \mathrm{~m}^{2} \mathrm{~g}^{-1}$ then with a current density of $c f .1 \mathrm{~A} \mathrm{~cm}_{\text {geo }}{ }^{-2}$ being drawn the molecular turnover rate of hydrogen per surface area of the catalyst will be $>1000 \mathrm{~nm}^{-2} \mathrm{~s}^{-1}$.

18. Stephens, I. E. L.; Rossmeisl, J.; Chorkendorff, I., Toward sustainable fuel cells. Science 2016, 354, 1378-1379.

19. Bu, L.; Zhang, N.; Guo, S.; Zhang, X.; Li, J.; Yao, J.; Wu, T.; Lu, G.; Ma, J.-Y.; Su, D., Biaxially strained $\mathrm{PtPb} / \mathrm{Pt}$ core/shell nanoplate boosts oxygen reduction catalysis. Science 2016, 354, 1410-1414.

20. Li, M.; Zhao, Z.; Cheng, T.; Fortunelli, A.; Chen, C.-Y.; Yu, R.; Zhang, Q.; Gu, L.; Merinov, B. V.; Lin, Z., Ultrafine jagged platinum nanowires enable ultrahigh mass activity for the oxygen reduction reaction. Science 2016, 354, 1414-1419.

21. Chen, C.; Kang, Y.; Huo, Z.; Zhu, Z.; Huang, W.; Xin, H. L.; Snyder, J. D.; Li, D.; Herron, J. A.; Mavrikakis, M., Highly crystalline multimetallic nanoframes with three-dimensional electrocatalytic surfaces. Science 2014, 343, 1339-1343.

22. Higher rates are generally not limited by the onset of turbulence but due to difficulties in fabrication of suitable high speed rotors

23. Masa, J.; Batchelor-McAuley, C.; Schuhmann, W.; Compton, R. G., Koutecky-Levich analysis applied to nanoparticle modified rotating disk electrodes: electrocatalysis or misinterpretation. Nano Res. 2014, 7, 71-78. 
24. Zhou, R.; Zheng, Y.; Jaroniec, M.; Qiao, S.-Z., Determination of the electron transfer number for the oxygen reduction reaction: from theory to experiment. ACS Catal. 2016, 6, 47204728.

25. Treimer, S.; Tang, A.; Johnson, D. C., A consideration of the application of Koutecký-Levich plots in the diagnoses of charge-transfer mechanisms at rotated disk electrodes. Electroanalysis 2002, 14, 165.

26. Zalitis, C. M.; Kramer, D.; Kucernak, A. R., Electrocatalytic performance of fuel cell reactions at low catalyst loading and high mass transport. Phys. Chem. Chem. Phys. 2013, 15, 43294340.

27. Jiao, X.; Tanner, E. E. L.; Sokolov, S. V.; Palgrave, R. G.; Young, N. P.; Compton, R. G., Understanding nanoparticle porosity via nanoimpacts and XPS: electro-oxidation of platinum nanoparticle aggregates. Phys. Chem. Chem. Phys. 2017, 19, 13547-13552.

28. Jiao, X.; Lin, C.; Young, N. P.; Batchelor-McAuley, C.; Compton, R. G., Hydrogen Oxidation Reaction on Platinum Nanoparticles: Understanding the Kinetics of Electrocatalytic Reactions via “Nano-Impacts”. J. Phys. Chem. C 2016, 120, 13148-13158.

29. Ly, L. S. Y.; Batchelor-McAuley, C.; Tschulik, K.; Kätelhön, E.; Compton, R. G., A critical evaluation of the interpretation of electrocatalytic nanoimpacts. J. Phys. Chem. C 2014, 118, 1775617763.

30. Zhi - peng, X.; Hai - qiang, D.; Pekka, P.; Zhi - yong, F.; Su - li, W.; Daniel, M.; Gong quan, S.; Zhen - xing, L., Electrochemical Dynamics of a Single Platinum Nanoparticle Collision Event for the Hydrogen Evolution Reaction. Angew. Chem. Int. Ed. 2018, 57, 3464-3468.

31. Kleijn, S. E. F.; Serrano-Bou, B.; Yanson, A. I.; Koper, M. T. M., Influence of hydrazineinduced aggregation on the electrochemical detection of platinum nanoparticles. Langmuir 2013, 29, 2054-2064.

32. Sokolov, S. V.; Tschulik, K.; Batchelor-McAuley, C.; Jurkschat, K.; Compton, R. G., Reversible or not? Distinguishing agglomeration and aggregation at the nanoscale. Anal. Chem. 2015, 87, 10033-10039.

33. Bond, A. M.; Fleischmann, M.; Robinson, J., Electrochemistry in organic solvents without supporting electrolyte using platinum microelectrodes. J. Electroanal. Chem. Interfacial Electrochem. 1984, 168, 299-312.

34. Li, X.; Batchelor - McAuley, C.; Laborda, E.; Compton, R. G., Aqueous Voltammetry in the Near Absence of Electrolyte. Chem. - Eur. J. 2017, 23, 15222-15226.

35. Batchelor-McAuley, C.; Ellison, J.; Tschulik, K.; Hurst, P. L.; Boldt, R.; Compton, R. G., In situ nanoparticle sizing with zeptomole sensitivity. Analyst 2015, 140, 5048-5054.

36. Broeke, J.; Perez, J. M. M.; Pascau, J., Image Processing with ImageJ. Packt Publishing: 2015.

37. Creighton, J. A.; Eadon, D. G., Ultraviolet-visible absorption spectra of the colloidal metallic elements. J. Chem. Soc., Faraday Trans. 1991, 87, 3881-3891.

38. Bobbert, P. A.; Wind, M. M.; Vlieger, J., Diffusion to a slowly growing truncated sphere on a substrate. Phys. A 1987, 141, 58-72.

39. Lin, C.; Jiao, X.; Tschulik, K.; Batchelor-McAuley, C.; Compton, R. G., Influence of Adsorption Kinetics Upon the Electrochemically Reversible Hydrogen Oxidation Reaction. J. Phys. Chem. C 2015, 119, 16121-16130.

40. Zalitis, C. M.; Kucernak, A. R.; Sharman, J.; Wright, E., Design principles for platinum nanoparticles catalysing electrochemical hydrogen evolution and oxidation reactions: edges are much more active than facets. J. Mater. Chem. A 2017, 5, 23328-23338.

41. Sheng, W.; Zhuang, Z.; Gao, M.; Zheng, J.; Chen, J. G.; Yan, Y., Correlating hydrogen oxidation and evolution activity on platinum at different $\mathrm{pH}$ with measured hydrogen binding energy. Nat. Commun. 2015, 6, 5848. 
42. Van der Niet, M. J. T. C.; Garcia-Araez, N.; Hernández, J.; Feliu, J. M.; Koper, M. T. M., Water dissociation on well-defined platinum surfaces: The electrochemical perspective. Catal. Today 2013, 202, 105-113.

43. Conway, B. E.; Tilak, B. V., Interfacial processes involving electrocatalytic evolution and oxidation of H2, and the role of chemisorbed H. Electrochim. Acta 2002, 47, 3571-3594.

44. The magnitude of the charge passed at $+0.2 \mathrm{~V}$ indicates that in comparsion to the expected charge for a single monolayer of $\mathrm{H}_{\text {upd }}$ that the average agglomerate size per impact is maximally 2 .

45. Apelblat, A.; Barthel, J., Conductance Studies on Aqueous Citric Acid. In Z. Naturforsch. A, 1991; Vol. 46, p 131.

46. A $25 \mathrm{~nm}$ long pore of radius $1 \mathrm{~nm}$ with a solution phase conductivity of $4.6 \mathrm{mS} \mathrm{m}^{-1}$ will have a resistance of approximately $1 \mathrm{~T} \Omega$, hence the passage of only tenths of a picoampere will lead to appreciable drops in potential along the structure.

\section{Table of Contents:}
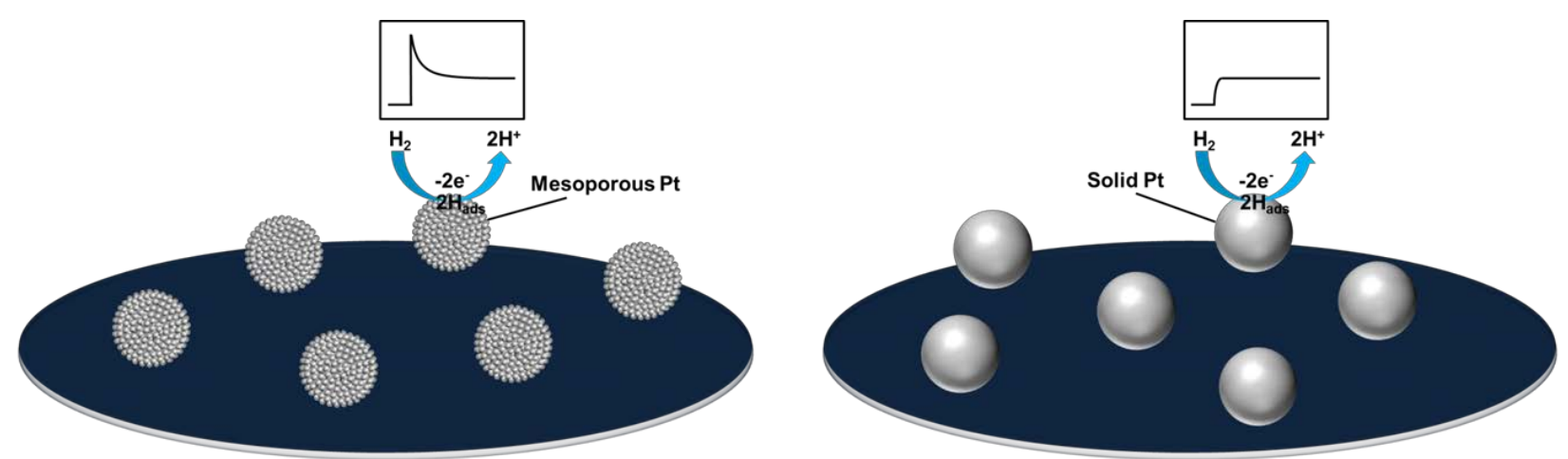